\title{
Emotions as a Communication Medium between the Unconscious and the Conscious
}

\author{
Matthias Rauterberg \\ Industrial Design, Eindhoven University of Technology \\ Den Dolech 2, 5600MB Eindhoven, The Netherlands \\ $\mathrm{g} \cdot \mathrm{w} \cdot \mathrm{m} . \mathrm{rauterberg@tue.nl}$
}

\begin{abstract}
Emotions are conceptualized and framed as a separate entity in human's cognitive architecture. In this paper I present the different idea that emotions are the form in which the result from a high dimensional optimization process happening in the unconscious is communicated to the low dimensional conscious. Instead of framing emotions as a separate sub component of our cognitive system, I argue for emotions as the main characteristic of the communication between the unconscious and the conscious. Based on this holistic view I recommend a different design and architecture for entertainment robots and other entertainment products with 'emotional' behavior. Intuition is the powerful information processing function of the unconscious while emotion is the result of this process communicated to the conscious. Emotions are the perception of the mapping from the high dimensional problem solving space of the unconscious to the low dimensional space of the conscious.
\end{abstract}

Keywords: emotion, intuition, unconscious, conscious, mapping.

\section{Introduction}

A lot of concepts and frameworks about emotions are already available. In this paper I argue for a new holistic view of the relation between the unconscious and the conscious information processing part of our brain. Although a lot of research has been done since the 80ties of last century [1], still important questions are unanswered [2]. The most accepted view on emotion is a modular sub component of our cognitive system linked and related to a lot of cognitive functions [3]. This conceptualization is also leading the design and architecture of entertainment robots [4]. I will argue for a different view so that designers of such kind of systems are better supported than nowadays.

One of the main characteristics of emotions is their richness, heterogeneity, vagueness and openness for multiple interpretations [5]. This is one of the most interesting but also often overlooked and underestimated aspect of emotions. I will argue for a new way of how to conceptualize emotions as a central aspect between the unconscious and the conscious information processing. 


\section{Emotion, Unconscious and the Mapping Problem}

In this chapter I discuss the state of art [1] and beyond for concepts about emotion, unconscious and mappings from high dimensional spaces to low dimensional spaces.

\subsection{Concepts of Emotions: The State of Art}

Several cognitive functions can be ordered according to their life-span (see Figure 1). Proper design of entertainment systems has the potential to stimulate and influence most of these functions. The primary cognitive functions are: reflexes, sensations, thoughts, dreams, emotions, moods, and drives (see also [1]).

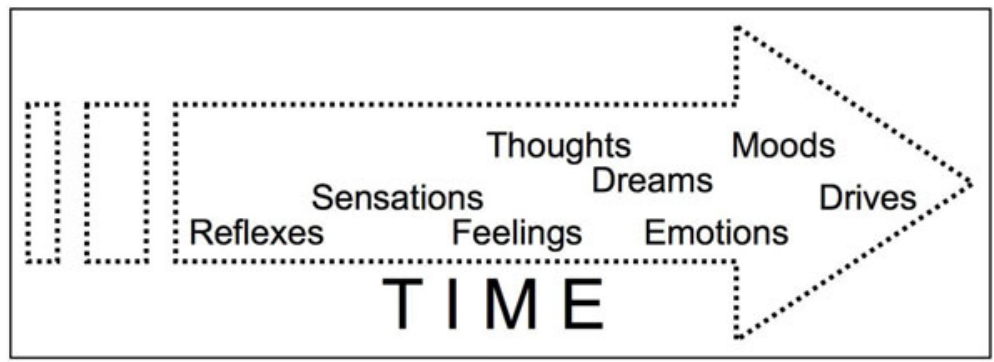

Fig. 1. Time scale of some cognitive functions (adopted from [6])

These different cognitive functions are linked to different control systems (bold black in Figure 2) of our body. In turn, these links help us design the right interaction (italic in Figure 2) through various body parts and control systems. To achieve users' emotional involvement, one needs to address these interactions with the right channels (as the examples given to the right of Figure 2).

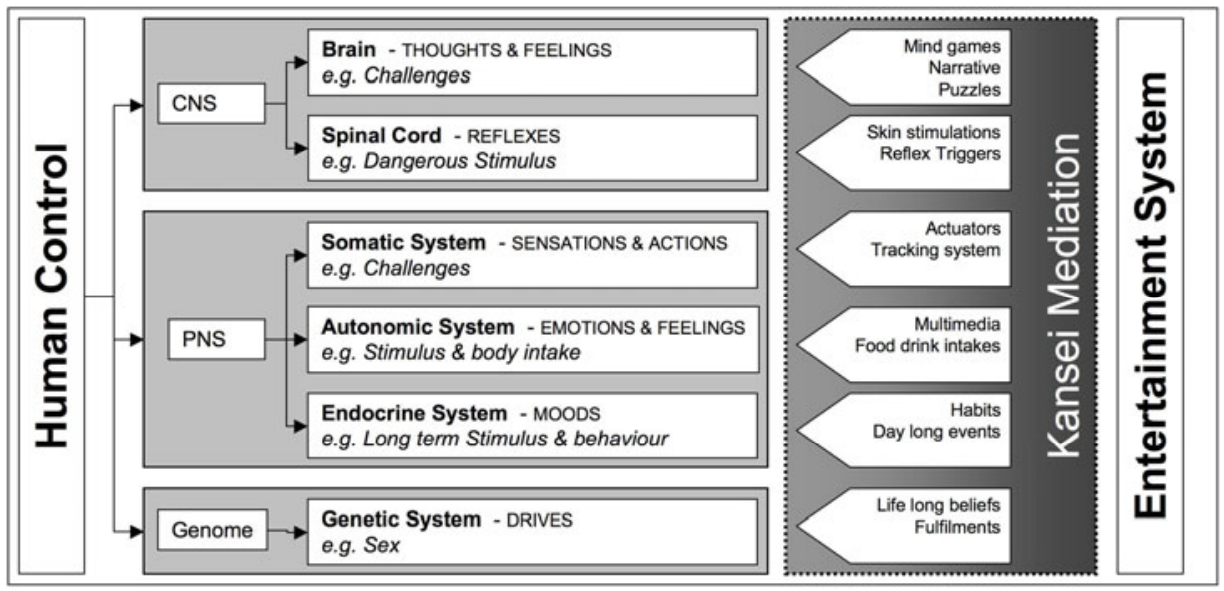

Fig. 2. From human control mechanisms to entertainment [CNS: Central Nervous System, PNS: Peripheral Nervous System] (adopted from [6]) 
The human unconscious can be framed by the genetic reproduction system, the peripheral nervous system (PNS) and the central nervous system (CNS). These three distinct systems contribute to unconscious information processing. Each of them has certain sub-systems which are directly related to emotional feelings (see Figure 2; [1]). One of the main differences between these subsystems is the cycle time to process incoming signals to actions executed (from milliseconds to hours; see Figure 1). All of these processes are related in one or the other way to emotions $[1,7]$.

Research in psychology has tried to structure prototypical emotional feelings into discrete basic categories [8-9]. Because emotions are complex, divers and with multiple facets there are different ways to construct these basic categories. The boundaries of the phenomenon emotion are so blurry that almost every feeling, mood and other internal perceptions can be categorized as an emotion. The phenomenon emotion is too broad to fit into one single scientific category. Emotions vary along certain dimensions (i.e. intensity, amount of pleasure, degree of activation, etc.).

One of the most prominent models is based on two dimensions: 'unpleasantpleasant' and 'activation-deactivation' as the core affect [10]. "Emotion categories do not cluster at the axes, and thus the structure of emotion has been said to be a circumplex. Nevertheless, although we are among those who emphasize such findings, we now believe this dimensional structure represents and is limited to the core affect involved. Prototypical emotional episodes fall into only certain regions of the circumplex" [8, p. 807] (see Figure 3).

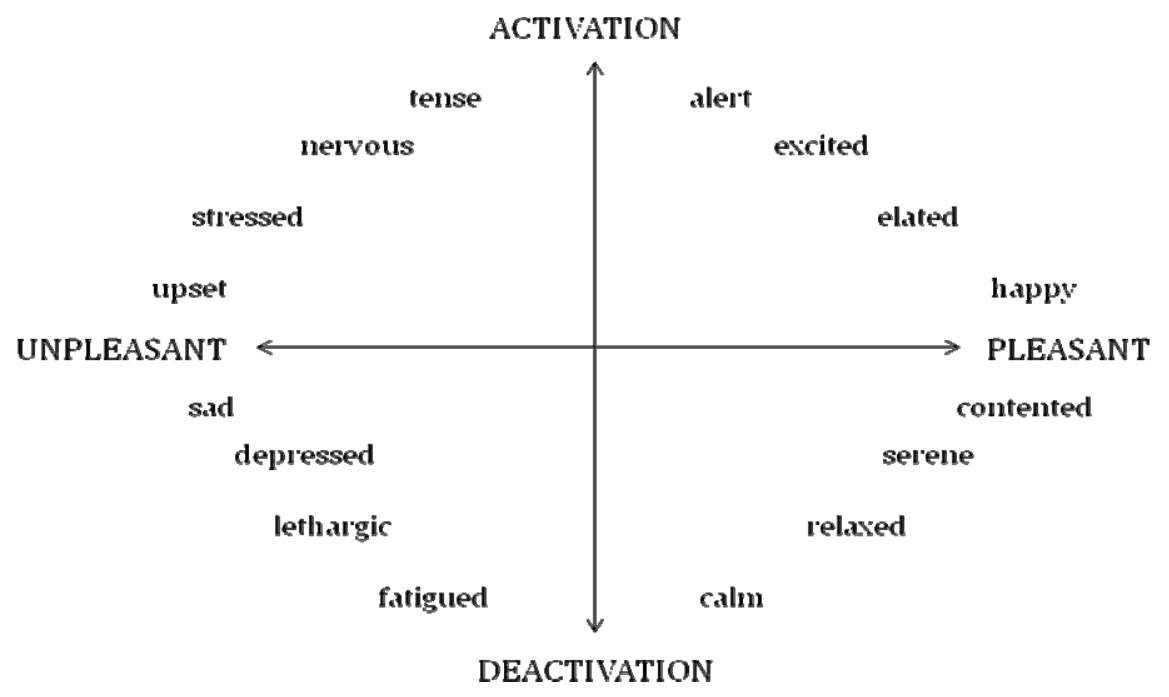

Fig. 3. The two dimensional core affect model (adapted from [11])

The combination of the two object-less dimensions 'pleasure-displeasure' and 'activation-deactivation' might capture most emotions but certainly not all. Cognitive information processing, intuition and behavioral planning can account for the myriad manifestations of emotions. The approach so far to capture emotions is dimensional 
and in addition limited. "The process of changing core affect is not fully understood, but the important point here is the complexity of the causal story" [11, p. 148].

Lane [12] goes even a step further by relating neural correlates to conscious emotional experience. He put forward a hierarchical model as follows [neuroanatomical structure/psychological function; however a one-to-one mapping between neuroanatomy and psychology is not intended]: brainstem/visceral activation, diencephalon/action tendencies, limbic/discrete emotion, paralimbic/blends of emotion, and prefrontal cortex/blends of blends. I can conclude that emotions can be based on conscious and unconscious information processing. Both processes have influences on actual behavior, behavior control and internal adaptation through learning.

Although emotions are complex phenomena and rich in content, main stream psychology tries to capture them in less complex models and frameworks. If we want to maintain the richness in our understanding of emotions, we probably have to change our view. Before I come back to this I will first introduce the unconscious cognitive functions.

\subsection{The Power of the Unconscious}

When we think of being conscious, we think of being awake and aware of our surroundings (see for more at [13]). Being conscious also means being aware of ourselves as individuals. Mostly, people tend to think of being conscious as being alive. We tend to think that the person should be responsive to the surrounding environment to be conscious. Being in a coma is considered to be the opposite of conscious, so called non-conscious or unconsciousness. There are at least three forms of consciousness for humans: (1) the conscious state; (2) the subconscious state; and (3) the unconscious state. In the scope of this paper the unconscious state is fully operational and functional for a normal human living as a parallel background process of our mind and body, we are just not aware of (e.g. activities of the cerebellum). The subconscious can be turned into conscious (i.e. by paying attention to subconscious activities); the unconscious normally is not available to the conscious. The remaining question is how -if at all- does the unconscious communicate with the conscious?

The conscious part of the brain is investigated already for a long time. One of the important results is the limitations of the information processing capacity of the short-term memory [14]. In his classical paper Miller [15] found that the conscious information processing capacity is limited to seven (plus or minus two) chunks or dimensions [16]. This conscious part is mainly described as the short-term or working memory to emphasize its role in decision making and controlling behavior.

Consciousness is a topic for which either there exist no acceptable description, definition and explanation or, and this depends on one's point of view, there are far too many and far too divergent ones. Most definitions from the Western world are resulting in Descartes dualism [17]. This dualism has created a schism between mind and body that does not necessarily exist and that has been a key, not necessarily a correct one, in the Western world understanding of consciousness. Even today's literature is full of reference to the mind and the body as if it has been established beyond doubt that there was indeed a separation [18]. The illusory Cartesian self is more and more challenged by biological and neurofunctional evidences that point to consciousness as an emergent property of competing and successive brain processes. Unconscious and 
conscious play a role in the initiation and performance of voluntary actions [19]. Both action and expression originate in the unconscious [20]. They are probably then vetoed by emotions and moods. Actions and expressions not vetoed are then performed.

In the dualist approach advocated by Descartes, the mind is conscious and the body unconscious. Outside cognitive and brain sciences was and still is a primacy of conscious over unconscious [1]. The unconscious activities of the human mind are hidden under and are controlled by consciousness (the word oppressed is often used). In the emergent view however, there is no such separation between mind and body and consciousness is said to be an emerging property of unconsciousness. It is moving away from the concept of conscious oppression of perception and expression into the concept of emerging perception and expression. Nakatsu, Rauterberg and Salem [19] show a model of the different views of the relationship between human consciousness and sub-consciousness, now (dualist view) and in the future (emergent view).

An iceberg can serve as a useful metaphor to understand the unconscious mind [21-22]. As an iceberg floats in the water, the huge mass of it remains below the surface. Only a small percentage of the whole iceberg is visible above the surface. In this way, the iceberg is like the mind. The conscious mind is what we notice above the surface while the sub- and unconscious mind, the largest and most powerful part, remains unseen below the surface. The unconscious mind holds all awareness that is not presently in the conscious mind. All activities, memories and thoughts that are out of conscious awareness are by definition sub- or even unconscious. Scherer [22] assumes that a large part of emotions functions in an unconscious mode and only some parts will emerge into conscious. But how does this relationship between unconscious and conscious look like?

Recent investigation in cognitive psychology of the conscious and unconscious are promising. Most of the brain's energy consumption is not used for processing responses to external stimuli as usually assumed; but what is this enormous amount of brain energy then for? [23-24] One promising aspect of unconscious information processing is finding optimal solutions in the multidimensional sensor and knowledge space of the unconscious for controlling behavior by situated forcasting the near and far future. But how does the unconscious communicate these 'solutions' to the conscious? Before I can provide an answer, I have to introduce the 'mapping problem' from a high (i.e. unconscious) to a low dimensional (i.e. conscious) processing space. The following chapter is pure metaphorical, all introduces concepts have no specific technical meaning in the context of this paper.

\subsection{Mapping from High to Low Dimensional Spaces}

A standard problem in several research areas is the visualization of results found in a high dimensional space into the two dimensional (2D) space of plane paper. This problem is quite old and several solutions are already developed, depending on the particular mapping problem [25-27]. To introduce into these approaches I will shortly discuss this classical 3D-2D example. Abbott [28] wrote the book 'Flatland'. Flatland has only two dimensions, and is populated with lines (females), triangles, squares, polygons, and circles; these inhabitants perceive their 2D environment differently than we do perceive our three dimensional (3D) environment. The basic idea is to explain the main differences about a fourth dimension beyond our 3D world. 
Flatlanders cannot understand a third dimension, and we have the same trouble with 4D, and definitively for higher dimensions. In Flatland for example is the ball from a 3D world perceived as changing diameters of circles, the 2D 'shadow' (see Figure 5).

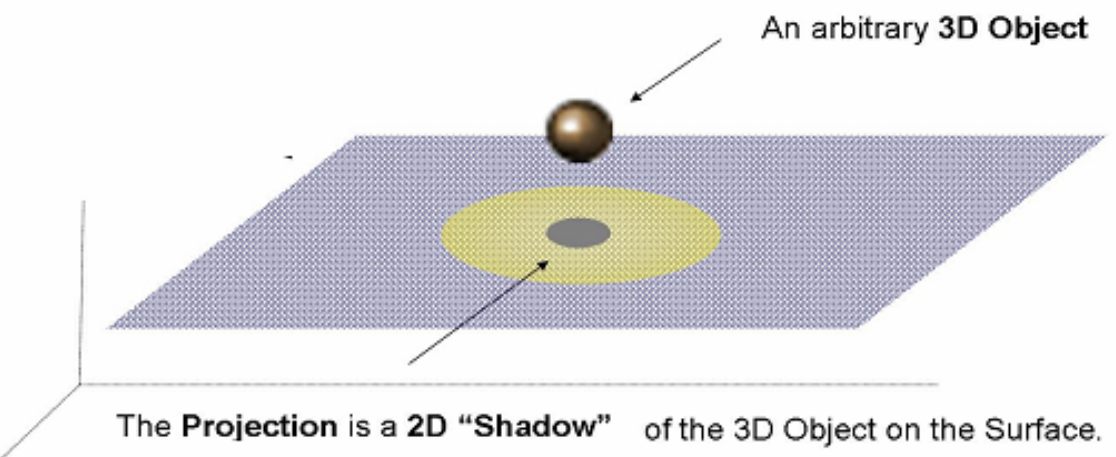

Fig. 4. The projection of a ball shaped $3 \mathrm{D}$ object on a $2 \mathrm{D}$ surface results in its form of a flat circle (from http://mdchristian.com/Normal_Projections.html)

Rucker [29] goes beyond Abbott's idea by taking the 2D world of the Flatlanders into curved space, black holes, and beyond. Banchoff [30] describes the problem of mappings between different dimensional spaces in more technical terms, applying several mapping methods to the design of computer graphics and other graphical shapes. He uses techniques of slices, projections, shadows, and generalization. The most practical part is learning to count the number of faces, vertices, and edges in a 4D (and higher) hypercube.

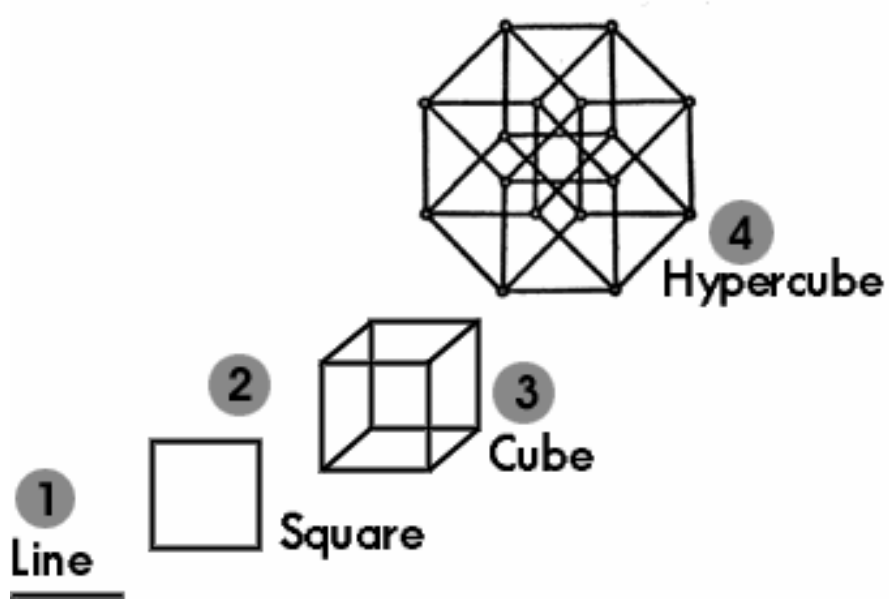

Fig. 5. Mapping a one dimensional line into two dimensions results in a square, a square into three dimensions in a cube, a cube into four dimensions in a hypercube (adopted from http://qunud.wordpress.com/) 
The mapping downwards into a three dimensional space of a hypercube results in eight different cubes. Figure 5 cannot exist in the real world with a $3 \mathrm{D}$ space; in addition Figure 5 itself is the projection of higher dimensional objects onto two dimensions of this 2D paper surface. If I now assume that the unconscious information processing often described as intuition [31-32] takes place in a high dimensional space, then I have to question how the solutions found in this high dimensional space can be mapped into a low dimensional space, the conscious reasoning?

\section{From Unconscious to Conscious}

Decades ago, Dreyfus questioned already the rational approach of cognitivism by excluding intuition, etc. [33]. Since then luckily a lot of research - in particular in psychology -have shown the growing interests in phenomena like tacit knowledge [34], intuition [31-32] and the unconscious [35]. Scherer assumes that a large majority of cognitive processes related to emotions are unconscious and "that only some of these processes (or their outcomes) will emerge into consciousness for some time" [22, p. 312]. First I have to show that the conscious and the unconscious are separated but related. Kahneman clearly operates on the assumption that both systems are distinct [36], and the unconscious is fallible. He describes the unconscious as 'intuition' and the conscious as 'reasoning'; both systems have clear complementary characteristics (see Table 1). Dienes and Scott conclude that the structural knowledge is really divided between the unconscious and the conscious [37].

Table 1. The two primary cognitive systems: intuition/unconscious and reasoning/conscious. (Adapted from [36, p. 1451])

\begin{tabular}{ll}
\hline INTUITION & REASONING \\
\hline Fast processing & Slow processing \\
Parallel processing & Serial processing \\
Automatic processing & Conscious controlled \\
Effortless & Effortful \\
Associative & Rule governed \\
Slow learning & Flexible, adaptive \\
Emotional / 'hot' & Neutral / 'cold' \\
\hline
\end{tabular}

Recent investigations in cognitive psychology of the nature of conscious and unconscious information processing are promising. Most of the brain's energy consumption is not used for processing responses to external stimuli; but what is this brain energy then for? [23-24]. One possible answer is for sub- or even unconscious information processing that guides behavior through situated forcasting. "Emotions are part of the biological solution to the problem of how to plan and carry out action aimed at satisfying multiple goals in environments which are not perfectly predictable" [10, p. 35]. According to the 'deliberation-without-attention' effect [38] it is not always advantageous to engage in intensive conscious decision making alone. On the basis of recent insights into the characteristics of conscious and unconscious thought, Dijksterhuis et al. [21] tested the hypothesis that simple choices produce 
better results after conscious information processing, but that choices in complex situations should be left to unconscious information processing. It was confirmed in several studies on consumer choice that purchases of complex products were viewed more favorably when decisions had been made in the absence of attentive deliberation. In addition, Dijksterhuis, Bos, Nordgren, and van Baaren [24, 39] could show the advantages of the unconscious information processing for complex processing, and therefore they open a door to a new view on the relationship towards the power of the unconscious [19, 38, 40]. Dienes and Scott showed in their experiments that conscious structural knowledge is associated with greater consistency in making errors than the unconscious [37, p. 348].

\section{Discussion and Conclusions}

The emotion experience and other cognitive activities are not separate, independent and distinctive processes, but should be conceptualized as a gradient in the interaction between cognitive activities (i.e. thoughts, memories, beliefs, etc.) [5]. "Brain structures at the heart of neural circuitry for emotion (e.g., the amygdale) impact cognitive processing from early attention allocation through perceptual processing to memory" [5, p. 390]. It seems not possible to explain how neural activities instantiates emotions if we conceptualize emotions as an independent cognitive process.

If we assume that emotions are perceived as important aspects in relation with other cognitive functions than we could go so far to conceptualize emotions as the appearance of these cognitive processes to our conscious. This is an internal perception loop about the own mental and bodily states [10,41]. If we assume further that the information processing capacity of the unconscious is several magnitudes higher than the conscious, and both systems are somehow separate systems, I have to answer the question how do these two systems communicate with each other. My idea is that emotions can play this role as the 'voice of the unconscious' in telling the conscious the solutions found in a high dimensional space. But these emotions are not only to inform the conscious, they also communicate to the social context around us. Our whole body language is also part of the emotional expression space for the adjustment of social relations [10].

Applying this view to the design of entertainment systems, in particular entertainment robots with human like behavior, I recommend implementing a high dimensional processing unit (mainly sensor data input related to models of the systems itself as of the environment) that maps the found solution of situated forecasting into a low dimensional action control unit, instead of implementing a separate emotion unit [34]. This mapping is 'colored' as emotions, primarily for external purposes in social communication expressed via the nonverbal behavior of the entertainment system.

\section{References}

1. Ortony, A., Clore, G.L., Collins, A.: The cognitive structure of emotions. Cambridge University Press, Cambridge (1988)

2. Ortony, A., Turner, T.J.: What's basic about basic emotions? Psychological Review 97, 315-331 (1990) 
3. Dörner, D., Hille, K.: Artificial souls: Motivated emotional robots. In: IEEE International Conference on Systems, Man and Cybernetics: Intelligent Systems for the 21st Century, pp. 3828-3832. IEEE Press, Piscataway (1995)

4. Itoh, K., et al.: Mechanisms and functions for a humanoid robot to express human-like emotions. In: Proceedings of the IEEE International Conference on Robotics and Automation, pp. 4390-4392. IEEE Press, Los Alamitos (2006)

5. Feldman-Barrett, L., et al.: The experience of emotion. Annual Review of Psychology 58, 373-403 (2007)

6. Salem, B., Nakatsu, R., Rauterberg, M.: Kansei mediated entertainment. In: Harper, R., Rauterberg, M., Combetto, M. (eds.) ICEC 2006. LNCS, vol. 4161, pp. 103-116. Springer, Heidelberg (2006)

7. Rolls, E.T.: Precis of The brain and emotion. Behavioral and Brain Sciences 23(2), 177-234 (2000)

8. Russell, J.A., Barrett, L.F.: Core affect, prototypical emotional episodes, and other things called emotion: Dissecting the elephant. Journal of Personality and Social Psychology 76(5), 805-819 (1999)

9. O'Rorke, P., Ortony, A.: Explaining emotions. Cognitive Science 18, 283-329 (1994)

10. Oatley, K., Johnson-Laird, P.N.: Towards a cognitive theory of emotions. Cognition and Emotion 1(1), 29-50 (1987)

11. Russell, J.A.: Core affect and the psychological construct of emotion. Psychological Review 110(1), 145-172 (2003)

12. Lane, R.D.: Neural correlates of conscious emotional experience. In: Lane, R.D. (ed.) Series in Affective Science, pp. 345-370. Oxford University Press, Oxford (2000)

13. Rauterberg, M.: Hypercomputation, unconsciousness and entertainment technology. In: Markopoulos, P., de Ruyter, B., IJsselsteijn, W.A., Rowland, D., et al. (eds.) Fun and Games 2008. LNCS, vol. 5294, pp. 11-20. Springer, Heidelberg (2008)

14. Baddeley, A.: The magical number seven: Still magic after all these years? Psychological Review 101(2), 353-356 (1994)

15. Miller, G.A.: The magical number seven, plus or minus two: Some limits on our capacity for processing information. The Psychological Review 63(2), 81-97 (1956)

16. Simon, H.A.: How big is a chunk? By combining data from several experiments, a basic human memory unit can be identified and measured. Science 183(4124), 482-488 (1974)

17. Descartes, R.: Discours de la méthode pour bien conduire sa raison, et chercher la vérité dans les sciences. Leiden, Ian Maire (1637)

18. Dreyfus, H.L., Dreyfus, S.E.: From Socrates to expert systems: The limits of calculative rationality. Technology in Society 6(3), 217-233 (1984)

19. Nakatsu, R., Rauterberg, M., Salem, B.: Forms and theories of communication: From multimedia to Kansei mediation. Multimedia Systems 11(3), 304-312 (2006)

20. Libet, B.: Can conscious experience affect brain activity? Journal of Consciousness Studies 10(12), 24-28 (2003)

21. Dijksterhuis, A., Nordgren, L.: A theory of unconscious thought. Perspectives on Psychology $1(2), 95-109$ (2006)

22. Scherer, K.R.: Unconscious processes in emotion. In: Feldman-Barrett, L., Niedenthal, P.M., Winkielman, P. (eds.) Emotion and Consciousness, pp. 312-334. Guildford, New York (2005)

23. Raichle, M.E.: The brain's dark energy. Science 314, 1249-1250 (2006)

24. Raichle, M.E.: A brief history of human brain mapping. Trends in Neurosciences 32(2), $118-126(2008)$

25. Tufte, E.R.: Envisioning information. Graphics Press, Cheshire (1990) 
26. Tufte, E.R.: The visual display of quantitative information. Graphics Press, Cheshire (1983)

27. Tufte, E.R.: Visual explanations. Graphics Press, Cheshire (1997)

28. Abbott, E.A.: Flatland: A romance of many dimensions. Dover Publications, Mineola (1992)

29. Rucker, R.: The fourth dimension: A guided tour of the higher universe. Houghton Mifflin, Boston (1984)

30. Banchoff, T.F.: Beyond the third dimension: Geometry, computer graphics, and higher dimensions. W.H. Freeman \& Company, New York (1996)

31. Khatri, N., Ng, H.A.: The role of intuition in strategic decision making. Human Relations 53(1), 57-86 (2000)

32. Witteman, C., et al.: Assessing rational and intuitive thinking styles. European Journal of Psychological Assessment 25(1), 39-47 (2009)

33. Dreyfus, H.L.: The Socratic and Platonic basis of cognitivism. AI \& Society 2, 99-112 (1988)

34. Reber, A.S.: Implicit learning and tacit knowledge. Journal of Experimental Psychology: General 118(3), 219-235 (1989)

35. Naccache, L., Dehaene, S.: Unconscious semantic priming extends to novel unseen stimuli. Cognition 80, 215-229 (2001)

36. Kahneman, D.: Maps of bounded rationality: Psychology for behavioral economics. The American Economic Review 93(5), 1449-1475 (2003)

37. Dienes, Z., Scott, R.: Measuring unconscious knowledge: distinguishing structural knowledge and judgment knowledge. Psychological Research 69, 338-351 (2005)

38. Dijksterhuis, A., et al.: On making the right choice: the deliberation-without-attention effect. Science 311, 1005-1007 (2006)

39. Coutrix, C., Nigay, L.: Mixed reality: A model of mixed interaction. In: Proceedings of International Working Conference on Advanced Visual Interface - AVI 2006, pp. 43-50. ACM, New York (2006)

40. Dehaene, S., et al.: Imaging unconscious semantic priming. Nature 395, 597-600 (1998)

41. Rauterberg, M.: About a framework for information and information processing of learning systems. In: Falkenberg, E.D., Hesse, W., Olivé, A. (eds.) Information System Concepts: Towards a Consolidation of Views, pp. 54-69. Chapman\&Hall, London (1995) 